\title{
A Pilot Study of Robot-Assisted Cochlear Implant Surgery Using Steerable Electrode Arrays
}

\author{
Jian Zhang ${ }^{1}$, Kai Xu ${ }^{1}$, Nabil Simaan ${ }^{1}$, Spiros Manolidis ${ }^{2}$ \\ Columbia University \\ ${ }^{1}$ Department of Mechanical Engineering \\ Laboratory of Advanced Robotics and Mechanism Applications \\ RM 234 S.W. Mudd Building, 500 West $120^{\text {th }}$ Street, New York, NY 10027, USA \\ $\left\{j z 2182, \mathrm{kx2} 001, \mathrm{~ns} 2236^{*}, @\right.$ columbia.edu $\}$ \\ http://www.columbia.edu/cu/mece/arma/ \\ ${ }^{2}$ Department of Otolaryngology - Head \& Neck Surgery \\ HP813, 180 Fort Washington Avenue, New York, NY 10032, USA \\ sm2307*@columbia.edu
}

\begin{abstract}
This paper presents results of a pilot study evaluating the efficacy of robotic assistance using novel steerable electrode arrays for cochlear implant surgery. The current surgical setup of cochlear implant surgery is briefly reviewed and its limitations are highlighted. In an effort to reduce trauma to the structure of the cochlea, the kinematics and path planning for novel cochlear steerable electrodes are developed to minimize the interaction forces between the electrode and the cochlea. An experimental robotic system is used to compare the electrode insertion forces of steerable implants with those of nonsteerable electrodes. The results of these experiments show about $70 \%$ reduction in the insertion forces when steerable electrodes are used with our proposed path planning and control. A distance metric explaining this reduction in the insertion force is defined and experimentally validated. Although this is only a preliminary study, we believe that these results provide a strong indication to the potential of robot-assisted cochlear implant surgery to provide a significant reduction in trauma rates during cochlear implant surgery.
\end{abstract}

\section{Introduction}

Cochlear implant surgery allows surgeons to restore partial hearing to patients suffering from severe hearing loss due to damaged neuroepithelial (hair) cells. During this surgery surgeons insert long, thin, and flimsy electrodes into the scala tympani canal. The interaction forces during this surgery are small (less than 10 grams) [1]. The tools used by surgeons do not provide any force feedback and are often very difficult to control. The complex anatomy of the cochlea does not lend itself to intraoperative imaging of its fine structures such as the basilar membrane. The basilar membrane is very delicate and can be easily ruptured by the inserted implant elec-

* Corresponding authors 
trode. All these characteristics of cochlear implant surgery currently limit both its success (lower than $60 \%$ of atraumatic insertions [2-4]) and applicability.

The success of an implanted electrode in restoring a wider frequency range depends on the depth of its atraumatic insertion. While increased depth of insertion promises improvement, it also increases the risk of trauma to the delicate structures of the cochlea. For these reasons cochlear implant surgery is currently characterized by shallow atraumatic insertions (less than $450^{\circ}$ ). Due to the traumatic nature of the electrode insertions cochlear implant surgery is currently not applicable to patients with residual hearing, but suffering from hearing loss that can not be restored by external hearing aids.

Following previous works on snake-like robots for distal dexterity enhancement $[5,6]$, this paper quantifies the potential improvement in cochlear implant surgery if robotic-assistance is used with novel steerable electrodes that provide some dexterity inside the cochlea. The design, kinematic modeling, calibration, and path planning of under-actuated flexible steerable electrodes are treated in this paper with a goal of minimizing the interaction forces with the walls of the cochlea. Mathematical modeling and path planning are presented in section 2. Although we present results using novel steerable electrodes currently being developed in our lab, our methodology in section 2 is valid for robotic assisted insertions using current electrodes with offstylet insertion tools [3]. These tools provide some control over the shape of the electrode while sacrificing force feedback due to the very small interaction forces and friction in the traditional insertion tools. Section 3 presents our experimental results comparing the insertion forces of straight non-steerable electrodes to steerable electrodes and quantifying the benefits of steerable implants.

\section{Problem statement and mathematical modeling}

The purpose of this preliminary study is to evaluate the efficacy of robot-assisted electrode insertion using novel steerable cochlear implant electrodes. We hypothesize that a reduction in the electrode insertion forces will result in a significant reduction in the trauma rates during cochlear implant surgery*. We thus use the insertion force of the electrode as a performance measure to quantify the potential benefits of using these steerable electrodes for reducing trauma in cochlear implant surgery.

Due to the small size of the cochlear implant electrodes we assume that controlling the shape of the electrode will be limited for a single actuator. Accordingly, we treat the steerable electrodes as continuum under-actuated snake-like robots. For these robots the solution of the direct kinematics is determined by the variational solution minimizing the elastic energy of the electrode $[7,8]$.

Figure 1 shows scaled up (3:1) steerable electrode models that we fabricated using silicone rubber. These implant models are actuated by a $\varnothing 100 \mu \mathrm{m}$ Kevlar thread. Figure 1-(b) and Figure 1-(c) show two different electrodes that we molded to yield different bending shapes. The problem at hand is to design an electrode that bends

\footnotetext{
- This hypothesis will be clinically tested in future studies
} 
into the desired shape that best approximates the shape of the cochlea and to determine the optimal path planning that will provide the best approximation of the shape of the cochlea during the electrode insertion process. This in turn will yield minimal interaction forces with the walls of the cochlea.

(a)

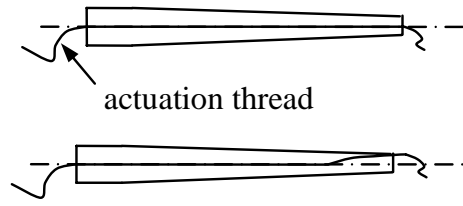

(b)

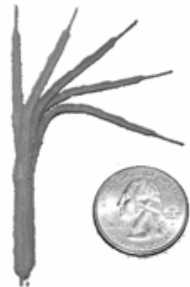

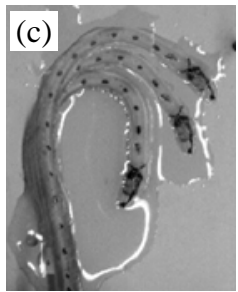

Figure 1. (a) Top and front view of the electrode, (b-c) Two electrode models with different bending characteristics

Direct kinematics of the steerable electrodes: Let $\theta$ and $s$ represent the angle and the arc length along the backbone of the electrode. Let $\mathrm{s}=0$ indicate the base and $\mathrm{s}=\mathrm{L}$ the tip of the electrode. Let $q$ be the value of the active joint controlling the bending of the electrode. The shape of a planar bending electrode is given by $\theta(s)$. Let the minimum energy solution for the direct kinematics of the electrode be approximated using a modal representation [9-12], Eq. (1) where vector a is a vector of modal factors. Let this vector of modal factors be given by $\mathbf{a}(q)=\mathbf{A} \boldsymbol{\eta}(q), \quad \boldsymbol{\eta} \in \mathfrak{R}^{m}, \mathbf{A} \in \mathfrak{R}^{n \times m}$ where $\boldsymbol{\eta}^{\mathrm{t}}=\left[1, \mathrm{q}, \mathrm{q}^{2}, \cdots, \mathrm{q}^{\mathrm{m}-1}\right]$. We note that for high-order polynomial approximations ( $m>6$ ), a set of orthogonal polynomials (e.g. Chebyshev polynomials) should be used for considerations of numerical stability [13].

$$
\theta(\mathrm{s}, \mathrm{q})=\boldsymbol{\psi}(\mathrm{s})^{\mathrm{t}} \mathbf{a}(\mathrm{q}), \quad \mathbf{a}, \boldsymbol{\psi} \in \mathfrak{R}^{\mathrm{n}} \text { where } \boldsymbol{\psi}(\mathrm{s})=\left[1, \mathrm{~s}, \ldots, \mathrm{s}^{\mathrm{n}-1}\right]^{\mathrm{t}}
$$

Since the minimum-energy solution does not lend itself to real-time control we choose to calibrate the direct kinematics experimentally. The shape of the electrode may be digitized by $r$ equidistant points along its backbone in $z$ different images of the electrode associated with $z$ different values of $q$. For each point along the backbone the angle of the curve tangent is digitized and recorded in an experimental data matrix $\boldsymbol{\Phi} \in \mathfrak{R}^{\mathrm{r} \times \mathrm{z}}$ such that $\Phi_{i, j}=\theta\left(s_{i}, q_{j}\right)$. Using the modal representation in Eq. (1) the direct kinematics problem is cast as an algebraic matrix equation, Eq. (2).

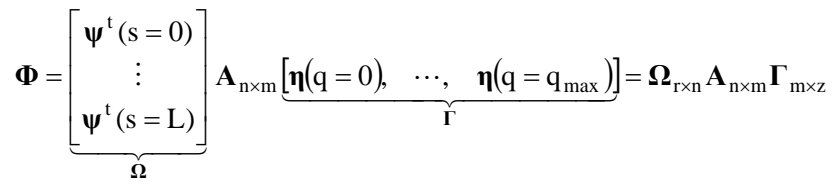

$$
\begin{aligned}
& \boldsymbol{\Omega}=\left[\begin{array}{cccc}
1 & s & \cdots & \mathrm{s}^{\mathrm{n}-1} \\
& & \vdots & \\
1 & \mathrm{~s} & \cdots & \mathrm{s}^{\mathrm{n}-1}
\end{array}\right]_{\mathrm{r} \times \mathrm{n}} \\
& \boldsymbol{\Gamma}=\left[\begin{array}{cccc}
1 & 1 & 1 \\
\mathrm{q} & \mathrm{q} & \cdots & \mathrm{q} \\
\vdots & \vdots & \vdots \\
\mathrm{q}^{\mathrm{m}-1} & \mathrm{q}^{\mathrm{m}-1} & \mathrm{q}^{\mathrm{m}-1}
\end{array}\right]_{\mathrm{m} \times \mathrm{z}}
\end{aligned}
$$


$\mathbf{\Omega}$ and $\boldsymbol{\Gamma}$ are Vandermonde matrices corresponding to the $r$ numerical values of $\mathrm{s}$ and the $z$ values of $q$ used to generate the experimental data matrix $\boldsymbol{\Phi}$. Solving Eq. (2) ( $\mathbf{\Phi}=\mathbf{\Omega A} \boldsymbol{\Gamma}$ ) for matrix $\mathbf{A}$ provides the required solution for the direct kinematics problem. The solution of this algebraic matrix equation is given by $\left[\boldsymbol{\Gamma}^{\mathrm{t}} \otimes \mathbf{\Omega}\right] \operatorname{Vec}(\mathbf{A})=\operatorname{Vec}(\boldsymbol{\Phi})$ [14]. Where $\otimes$ represents Kronecker's matrix product and $\operatorname{Vec}\left(\mathbf{A}_{m \times n}\right)=\left[a_{11} \cdots a_{m 1}, a_{12} \cdots a_{m 2}, a_{1 n} \cdots a_{m n}\right]^{t}$.

Optimal electrode insertion path planning: Let $s_{q}$ represent the electrode insertion depth and let $\theta_{c}(s)$ be the shape of the cochlea. Equation (3) returns the optimal value of $q$ that minimizes the shape difference between the inserted portion of the electrode and the cochlea. The optimal value of $q$ is found by calculating the objective function for all columns of $\boldsymbol{\Phi}$ and the minimum is found by numerical interpolation between the columns that best approximate the minimum value of the objective function.

$$
\underset{q}{\arg \min } \int_{L-s_{q}}^{L}\left(\left|\theta_{c}(s)-\theta(s)\right|^{2}\right)
$$

Figure 2 shows the results of a Matlab simulation of this path planning process applied using the experimental data matrix $\boldsymbol{\Phi}$ of the electrode of Figure 1-(c). The figure demonstrates that the implant coincides with the cochlear model well except for the tip because the implant does not actively bend at its tip.
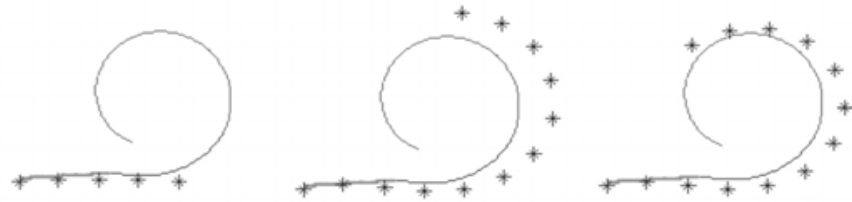

* = Indicates the shape of steerable electrode.

Solid line $=$ shape of the cochlea using $2 \mathrm{D}$ Cohen's template up to $330^{\circ}$ insertion angle

Figure 2. Path planning insertion simulation for the electrode in Figure 1-(c)

\section{Experimental validation}

Phantom models of the cochlea: The cochlea has a 3-dimensional spiral anatomical structure that was statistically characterized in the work of Cohen, et. al. [15], and extended to 3D by Ketten et. al. [16]. The backbone curve of the cochlea is given by Eq. (4) where $r, z$, and $\theta$ are the cylindrical coordinates of this curve ( $r$ is the radial distance to the curve, $z$ is the height, and $\theta$ is the angle). The values of the constants $a, c, b, d, \theta_{0}, p$ are based on [16], [17].

Figure 3-(a-b) show a CAD model of the cochlea and the scala tympani. Figure 3(c) shows a 3-dimensional stereo lithography 2:1 model of scala tympani. Figure Figure 3-(d) shows a 2-dimensional 3:1 scaled up model of the cochlea using Cohen's 
2D template. The 2-dimensional phantom model in Figure 3-(d) was used in this work for electrode insertion evaluation. Although this model does not provide insertion angles larger than $340^{\circ}$, it is enough to demonstrate the differences between passive electrodes and active bending electrodes used in our work.

$$
\theta \in\left[10.3^{\circ}, 910^{\circ}\right], \quad R=\left\{\begin{array}{cc}
c\left(1-d \log \left(\theta-\theta_{0}\right)\right) & \theta<100^{\circ} \\
a e^{-b \theta} & \theta \geq 100^{\circ}
\end{array}\right\} \quad, \quad z=p\left(\theta-\theta_{0}\right)
$$

A two Degrees-of-Freedom (DoF) experimental robotic insertion system with force monitoring: The experimental system shown in Figure 4 was used to compare electrode insertion forces for steerable and non-steerable electrodes. This system is composed from a single DoF linear actuator, AG NTEP 5000d single axis load cell, and a steerable implant that is inserted into the 3:1 phantom model of Figure 3-(d). The electrode model is supported against buckling using an implant support ring that is affixed to the force sensor. This support ring was placed in two axial positions: one for shallow insertions, and another for deep insertions, Figure 5. Since the direct kinematics of the implant is affected by the constraint imposed by the slip ring, we calibrated the direct kinematics for both configurations in Figure 5.

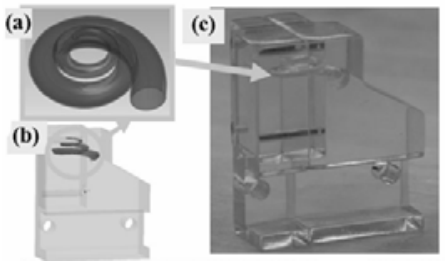

Figure 3. (a) A 3D CAD model of the cochlea. (b) stereo-lithography model of the scala tympani. (d) A planar 3:1 model of the cochlea
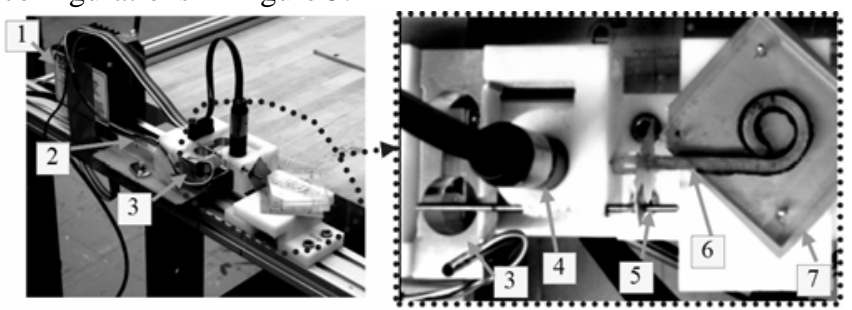

1. amplifier

2. linear actuator

3. force sensor

4. electrode actuator

5. implant support

6. implant

7. 2D cochlea model

Figure 4. Experimental system setup for robot-assisted electrode insertion

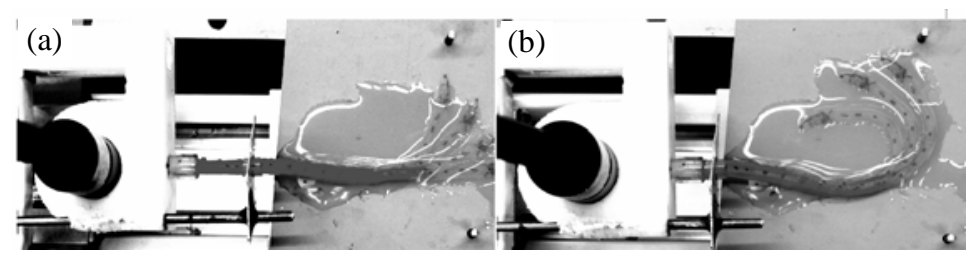

Figure 5. Two configurations of the implant buckling support ring were used: (a) Configuration for shallow insertion. (b) Configuration for deep insertion 
Direct kinematics calibration: For the electrode direct kinematics calibration we marked the electrode with sixteen equidistant points along its backbone. Twelve images with different total bending angles of the electrode were acquired and the position of the implant support ring was changed from a pre-set value for shallow insertions to another pre-set value for deep insertions, Figure 5.

Insertion experiments: The same electrode was inserted three times without controlling its bending (the electrode was set in a straight shape outside of the cochlea). Another set of three insertions was performed while controlling the bending of the electrode based on the path planning algorithm of section 2. As in [1], we wetted the cochlea with glycerin to emulate the friction conditions during surgery. The forces of insertion were recorded as a function of the insertion depth of the electrode.

Results: Figure 6-(a) shows the average insertion forces over three insertion experiments using the electrode of Figure 1-(c) with and without control of its bending. Figure 6-(b) compares the best insertion of the electrode without bending control with the three insertion experiments using the path planning of section 3 . The results show that the active bending insertions are repeatable and that the best non-steerable insertion is significantly worse than the worst steerable insertion. A prominent reduction of $\mathbf{6 8 \%}$ in the insertion force was achieved when using our path planning.
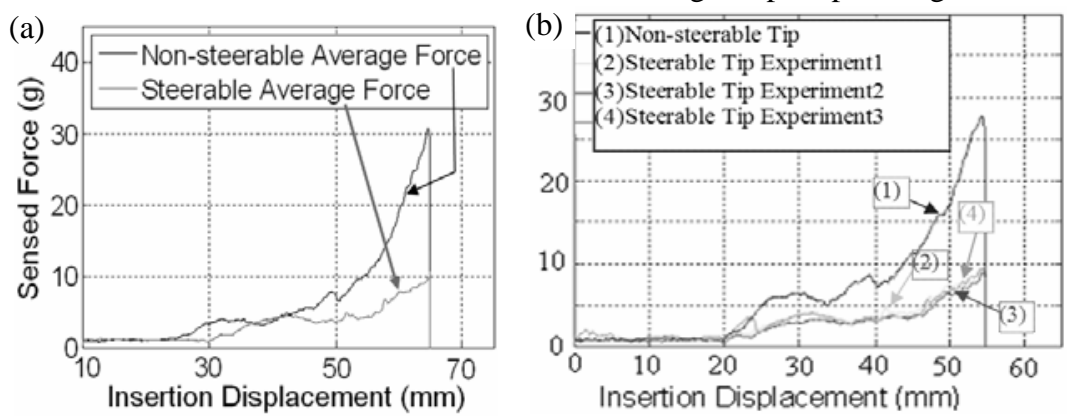

Figure 6. (a) Average insertion forces of steerable vs. non-steerable electrode over three insertions. (b) The insertion forces for all the steerable electrode insertions are significantly lower than the best non-steerable implant insertion

Analyzing experimental results: The insertion force due to friction between the electrode and cochlea is equivalent to friction force in a band brake system [18], which depends on the contact angle of the electrode with the external walls of the cochlea. To explain the results of Figure 6, several images during the insertion experiments were acquired for several insertion depths and digitized as shown in Figure 7-(a). Third-order polynomials were fitted to the digitized data to represent the curve of the external wall of the electrode, $\mathbf{r}_{C}$, and the curve of the outer wall of the cochlea , $\mathbf{r}_{I}$, Figure 7-(b). Using these polynomial representations a distance metric $e(\theta)=\left\|\mathbf{r}_{c}(\theta)-\mathbf{r}_{I}(\theta)\right\|_{2} \quad \theta \in[0, \varphi]$ was calculated (where $\varphi$ is the insertion angle) and averaged for every insertion angle during the insertion, Eq. (5). 


$$
\overline{\mathrm{e}}=\varphi^{-1} \int_{0}^{\varphi} e(\theta) d \theta
$$
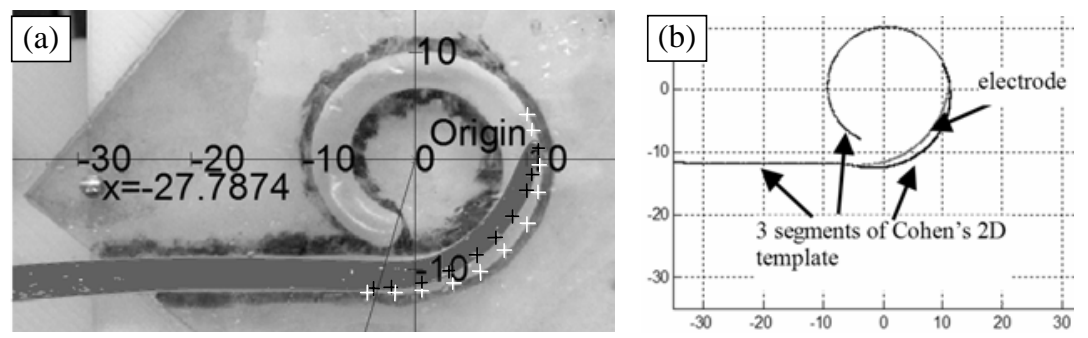

Figure 7. (a) A sample digitized image. (b) The plots of Cohen's 2D template and the curve of the outside wall of the electrode (all units in $\mathbf{m m}$ ).

Figure 8 shows the average distance metric $\overline{\mathrm{e}}$ vs. the electrode insertion depth. Figure 8 and Figure 6 explain the decrease in the insertion forces when the electrode is actuated since the average distance metric is increased significantly compared to the non-steerable electrode. Moreover, the difference between steerable and non-steerable electrodes becomes more prominent as the insertion depth increases.

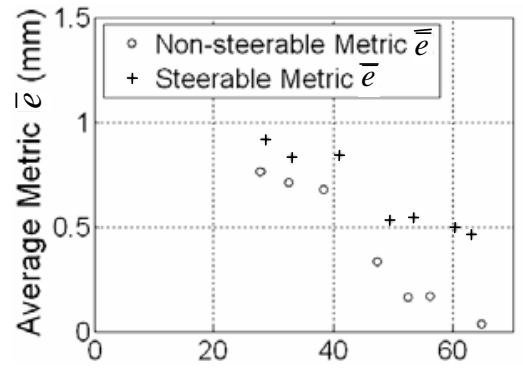

Figure 8. Average distance metric $\overline{\mathrm{e}}$ for Non-steerable and Steerable implant as a function of insertion depth (in mm)

\section{Conclusion}

This paper presented a pilot study that evaluates the potential benefits of robotassisted cochlear implant surgery using active-bending electrodes. Although this is a preliminary study using phantom models, it clearly indicates the potential of robotassisted cochlear implant surgery using steerable electrodes in reducing the trauma to the cochlea. The paper presented the mathematical modeling for the steerable electrodes including a path planning algorithm that minimizes the interaction forces during electrode insertion. An experimental system was presented and the results of insertion experiments comparing insertions using steerable versus non-steerable electrodes showed a reduction of about $70 \%$ in the insertion forces when steerable electrodes are used. The paper also correlated the reduction in the insertion forces with an increase in the average distance metric between the electrode and the cochlea as a result of the distal dexterity provided by the proposed steerable electrodes. Based on these results we are currently developing a tele-robotic system for robot-assisted cochlear implant surgery. 


\section{References}

1. Roland, T., A model for cochlear implant electrode insertion and force evaluation: results with a new electrode design and insertion technique. The Laryngeoscope, 2005. 115(august): p. 1325-1339.

2. Eshraghi, A., N. Yang, and T. Balkany, Comparative Study of Cochlear Damage with Three Perimodiolar Electride Designs. The Laryngeoscope, 2003. 113: p. 415-419.

3. Wardrop, P., et al., A temporal bone study of insertion trauma and intracochlear position of cochlear implant electrodes I: comparison of Nucleus banded and Nucleus Contour electrodes. Hearing Research, 2005. 203: p. 54-67.

4. Wardrop, P., et al., A temporal bone study of insertion trauma and intracochlear position of cochlear implant electrodes II: comparison of spiral clariontrade mark and HiFocus IItrade mark electrodes banded and Nucleus Contour electrodes. Hearing Research, 2005. 203: p. 68-79.

5. Wei, W., K. Xu, and N. Simaan. A compact Two-armed Slave Manipulator for Minimally Invasive Surgery of the Throat. in The first IEEE / RAS-EMBS International Conference on Biomedical Robotics and Biomechatronics (BIOROB'2006). 2006. Pisa, Italy.

6. Ikuta, K., K. Yamamoto, and K. Sasaki. Development of Remote Microsurgery Robot and New Surgical Procedure for Deep and Narrow Space. in IEEE International Conference on Robotics and Automation. 2003.

7. Walker, I. Some Issues in Creating "Invertebrate" Robots. in In the Proceedings of the International Symposium on Adaptive Motion of Animals and Machines. 2000. Montreal, Canada.

8. Gravagne, I. and I. Walker. On the Kinematics of Remotely-Actuated Continuum Robots. in IEEE International Conference on Robotics and Automation. 2000.

9. Chirikjian, G.S. and J.W. Burdick, A Modal Approach to Hyper-Redundant Manipulator Kinematics. IEEE Transactions on Robotics and Automation, 1994. 10(3): p. 343-354.

10. Burdick, J.W. and G. Chirikjian, The Kinematics of Hyper-Redundant Robots. The IMA Volumes in Mathematics and its Applications, ed. J. Baillieul, S. Sastry, and H. Sussmann. Vol. 104. 1998: Springer-Verlag.

11. Mochiyama, H. and H. Kobayashi. The Shape Jacobian of a Manipulator with Hyper Degrees of Freedom. in IEEE International Conference on Robotics and Automation. 1999.

12. Mochiyama, H., E. Shimemura, and H. Kobayashi. Shape Correspondence between a Spatial Curve and a Manipulator with Hyper Degrees pf Freedom. in IEEE/RSJ International conference on Intelligent Robots and Systems (IROS '). 1998.

13. Angeles, J. and C. Lopez-Cajun, Optimization of cam mechanisms. 1991: Kluwer Academic Publishers.

14. Lancaster, P. and L. Rodman, Algebraic Riccati Equations. 1995: Oxford Science Publications.

15. Cohen, L., et al., Improved and Simplified Methods for Specifying Positions of the Electrode bands of a Cochlear Implant Array. The American Journal of Otology, 1996. 17: p. 859-865.

16. Ketten, D.R., et al., In vivo measures of cochlear length and insertion depth of Nucleus cochlear implant electrode arrays. Ann. Otol. Rhinol. Laryngol., 1998. 107(12): p. 1-16.

17. Yoo, S.K., et al., Three-Dimensional Modeling and Visualization of the Cochlea on the Internet. IEEE Transactions on Information Technology in Biomedicine, 2000. 4(2): p. 144-151.

18. Juvinall, R. and K. Marshek, Fundamentals of Machine Component Design. 3rd ed. 2003: John Wiley \& Songs. 